\title{
Arrhythmic adult ecdysis but rhythmic emergence from the host chorion in Trichogramma embryophagum (Hymenoptera: Trichogrammatidae)
}

\author{
Sergey Ya. REZNiK, Natalia D. VOINOVich, Nina P.VAGHinA and Svetlana G. KaRPOVA
}

Zoological Institute, Russian Academy of Sciences, 199034 St. Petersburg, Russia; e-mail: reznik@MD12306.spb.edu

Key words. Parasitoids, Hymenoptera, Trichogramma, circadian rhythms, photoperiod, pupal-adult ecdysis, adult emergence

\begin{abstract}
In many insect species with a pupa covered by various "shells" (puparium, host remains, etc.) pupal-adult ecdysis and emergence to the open air represent two discrete steps. However, in Trichogramma, as well as in other insect parasitoids, these two processes have never been studied separately. We investigated the temporal pattern of pupal-adult ecdysis and of adult emergence from the host chorion in Trichogramma embryophagum Hartig (Hymenoptera: Trichogrammatidae) in laboratory conditions (12L : $12 \mathrm{D}, 20^{\circ} \mathrm{C}$ ). Adult ecdysis was arrhythmic, while adult emergence showed a strong rhythmicity. The time lag between ecdysis and emergence varied from one to almost two days, depending on the circadian time of the ecdysis. The proportion of ecdysed adults that stayed in the host chorion ranged up to $60 \%$ (just before the highest peak of emergence). The cumulative percentage of ecdysed adults gradually increased with time, independently of whether the light was turned on in accordance with the entrained circadian rhythm or $4 \mathrm{~h}$ earlier. This arrhythmic ecdysis could be explained by the fact that the ecdysed adults get into a well protected space inside the host chorion and the timing of this event is adaptively neutral.
\end{abstract}

\section{INTRODUCTION}

All living organisms are exposed to rhythmic environmental changes. As an adaptation to the oscillating environment, biological rhythms restricting various activities to the most favorable periods were acquired. The proper timing is particularly important for so called "once-in-alifetime" events and for the most vulnerable life stages, when each error could be fatal. Insect adult emergence combines these features. Therefore, the daily rhythm of this activity is usually regulated by a precisely adjusted mechanism so that eclosion is restricted to a very narrow period of day, termed as "a gate" (see review: Saunders, 2002).

Species of the genus Trichogramma Westwood (Hymenoptera: Trichogrammatidae) are minute egg parasitoids widely used for biological control of different insect pests in agriculture and forestry (Smith, 1996) and also as model insects for various researches. Particularly, distinct rhythms of adult emergence have been well characterized and intensively studied. It was repeatedly demonstrated that Trichogramma species possess circadian rhythms of adult emergence which could be synchronized with photo- or thermoperiod (Corrigan et al., 1995; Pompanon et al., 1995; Zaslavski et al., 1995; Zinovjeva et al., 1996; Doyon \& Boivin, 2005). The rhythms persisted with a period close to $24 \mathrm{~h}$ after transfer to aperiodic conditions (constant darkness and constant temperature), suggesting they were under the control of endogenous circadian oscillators (Dahiya et al., 1993; Karpova, 2006). However, direct influence of a light-on and/or a temperature step-up can induce emergence of a considerable fraction of adults almost at any moment of the circadian time, suggesting a strong exogenous (masking) effect (Zaslavski et al., 1999; Karpova \& Reznik, 2002; Kar- pova, 2006). Similar results were obtained for other egg parasitoids (Fantinou et al., 1998).

Noteworthy, in all these studies, as well as in some other publications (Saunders, 2002), the terms "ecdysis", "eclosion" and "emergence" were often used as synonyms, which is true only for certain insects with a free pupa. But in many other insects the pupa is covered by a shell: puparium, cocoon, host remains (as in Trichogramma and many other endoparasitoids), etc. Hence, the pupal-adult ecdysis and the emergence to the open air represent two discrete steps characterized by specific physiological and behavioral reactions (Reynolds, 1980). In many cases, particularly, in such well-known model insects as brachyceran Diptera (Drosophila, Calliphora, etc.) and in many moths (Saturniidae, Sphingidae, etc.) ecdysis is immediately followed by emergence (Reynolds, 1980; Baker et al., 1999) and therefore daily rhythms of these events practically coincide. However, in Trichogramma, as well as in other parasitic Hymenoptera emerging from the host at the adult stage, these two processes (as far as we know) have never been studied separately.

The aim of the present study was to investigate separately rhythms of adult ecdysis and of adult emergence from the host chorion in Trichogramma embryophagum Htg. under constant photoperiod and under anticipatory terminated scotophase.

\section{MATERIAL AND METHODS}

\section{General methods}

In our study, we used a parthenogenetic laboratory strain of $T$. embryophagum originally reared from Tortricidae eggs collected in Moscow province of Russia and then cultivated for more than 200 generations on the eggs of the Angoumois grain moth, Sitotroga cerealella Olivier (Lepidoptera: Gelechiidae). 
All wasps used in our experiments were reared in thermostatic chambers at $20 \pm 0.3^{\circ} \mathrm{C}$ under a photoperiod of $12 \mathrm{~L}: 12 \mathrm{D}$ and ca $70 \% \mathrm{RH}$. To start an experiment, a block of $12-15$ paper cards $(4 \times 10 \mathrm{~mm}$ each) was placed in a large $(2 \times 20 \mathrm{~cm})$ test tube with several hundred Trichogramma females. About $100 \mathrm{~S}$. cerealella eggs were pasted to each card and the wasps were allowed to parasitize the hosts during $2 \mathrm{~h}$. Then the cards were separated and placed in small test tubes for the wasps development and emergence under the same regime. Cards of the same block were considered as a replicate.

Trichogramma adult ecdysis from the pupal cuticle cannot be directly recorded through the chorion of the host egg. Therefore, the method used in the earlier studies (Zaslavski et al., 1995, 1999; Karpova \& Reznik, 2002; Karpova, 2006), i.e., periodic counting of emerged individuals, was no longer feasible. Thus, a new method was elaborated to monitor the temporal patterns of the processes under study: at regular intervals, one card was randomly selected from the cohort and immediately placed in a freezer $\left(-18^{\circ} \mathrm{C}\right)$. Then, frozen eggs were dissected and three parameters were separately recorded for each card: (1) the number of pupae, (2) the number of ecdysed adults inside host chorions, and (3) the number of emerged adults. Ecdysed individuals were easily distinguished from pharate adults (still covered with the pupal cuticle) by free (not fastened) head, legs, and antennae. In addition, pupal cuticle itself could be clearly seen, particularly around the neck region. The number of emerged adults was estimated by the number of blackish colored host eggs with emergence holes, because Trichogramma females usually lay only one egg in small hosts, such as $S$. cerealella. The dissections were conducted under a dissecting microscope, visual comparison of ecdysed and emerged adults was made with a transmission light microscope.

\section{Experimental design}

The first experiment was aimed at describing the general temporal pattern of the studied processes. During this experiment, samples (cards with host eggs) were taken every $6 \mathrm{~h}$ starting from the moment of the light-on two days before the first adults emergence and finishing at the end of the 4th day of emergence (at $20^{\circ} \mathrm{C}$, first adults emerged at the 18th day after the parasitization). A total of 360 cards with 39,360 parasitized host eggs were used in 24 replicates of this experiment.

The second experiment was conducted at a finer time scale. The samples were taken with intervals of $2 \mathrm{~h}$ from the end of the photophase during which the first emerged adults were recorded till the end of the next photophase. Thus, 12 samples were taken in each of 21 replicates of this experiment (with a total of 24,172 parasitized host eggs).

The third experiment also included 12 samples taken over 24 $\mathrm{h}$ at 2-hours-long intervals and the wasps developed under the same conditions. However, in contrast to the second experiment, in the day when records were made, the light onset was advanced by being turned on $8 \mathrm{~h}$ after the beginning of the scotophase (i.e., $4 \mathrm{~h}$ before the end of the scotophase in the second experiment). A total of 24,707 parasitized eggs were studied in 21 replicates of this experiment.

\section{Statistical treatment}

Each sample (card with the host eggs) was considered as an experimental unit. The cumulative percentage of adults emerged from the host and the cumulative percentage of ecdysed adults (including both emerged and stayed in the host chorion) were calculated for each sample. The intensity of emergence and the intensity of ecdysis were estimated by the difference between the percentages of, correspondingly, emerged and ecdysed adults between a given sample and a previous sample of the same replicate. Before the statistical analysis, percentages were arcsine - square root transformed and then treated with ANOVA. Untransformed data (medians and quartiles) were used as descriptive statistics both in the text and in the figures. All statistical procedures were calculated with SYSTAT 10.2.

\section{RESULTS}

The 1st experiment did not reveal any periodicity of adult ecdysis: the cumulative percentage of adults (Fig. 1A) progressively increased during 4 days. The ecdysis intensity peaked at the 3rd day of recording (Fig. 1B), when approximately half of the adults ecdysed. Adult emergence, on the contrary, showed a strong rhythmicity with a peak after light-on (Fig. 1B). There was a considerable time lag between ecdysis and emergence of the parasitoids. The duration of this period roughly estimated from the horizontal distance between the two cumulative curves varied from one to almost two days, depending on the circadian time of the ecdysis. As clearly seen from Fig. 1A, this time lag was maximal just before the peak of emergence and minimal at the level of a peak: about 22, 27,24 , and $20 \mathrm{~h}$ for the four successive peaks of emergence, correspondingly.

The proportion of ecdysed adults that stayed in the host chorion (the vertical distance between the two curves in Fig. 1A) was very high: up to $69 \%(66-76 \%)$ before the highest peak of emergence (hereafter, medians and quartiles are given). Interestingly, after the peak of mass emergence, the percentage of ecdysed adults that stayed in the host chorion was still high: $57 \%(44-61 \%)$ at $60 \mathrm{~h}$, $36 \%(30-43 \%)$ at $84 \mathrm{~h}$, and $12 \%(7-20 \%)$ at $108 \mathrm{~h}$ (Fig.

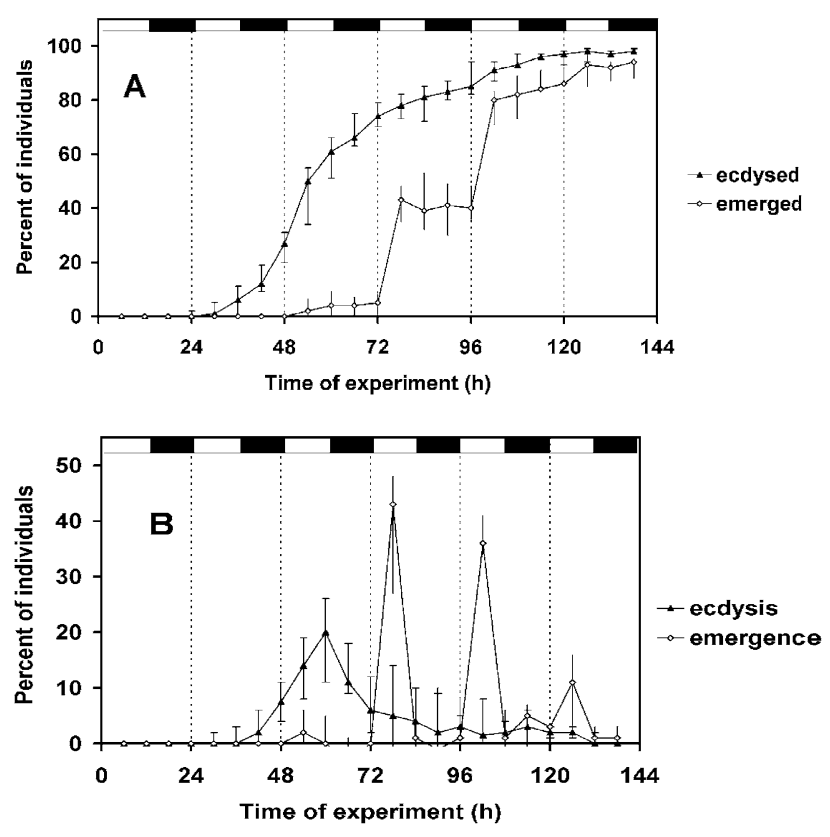

Fig. 1. Temporal patterns of adult ecdysis and emergence in Trichogramma embryophagum (the 1 st experiment). A - cumulative percentages of ecdysed and of emerged individuals; B ecdysis and emergence incidence at each time point. Medians and quartiles are shown, $n=24$. White and black bars above graphs indicate light and dark phases of photoperiod, vertical dashed lines indicate the moments of lights-on. 

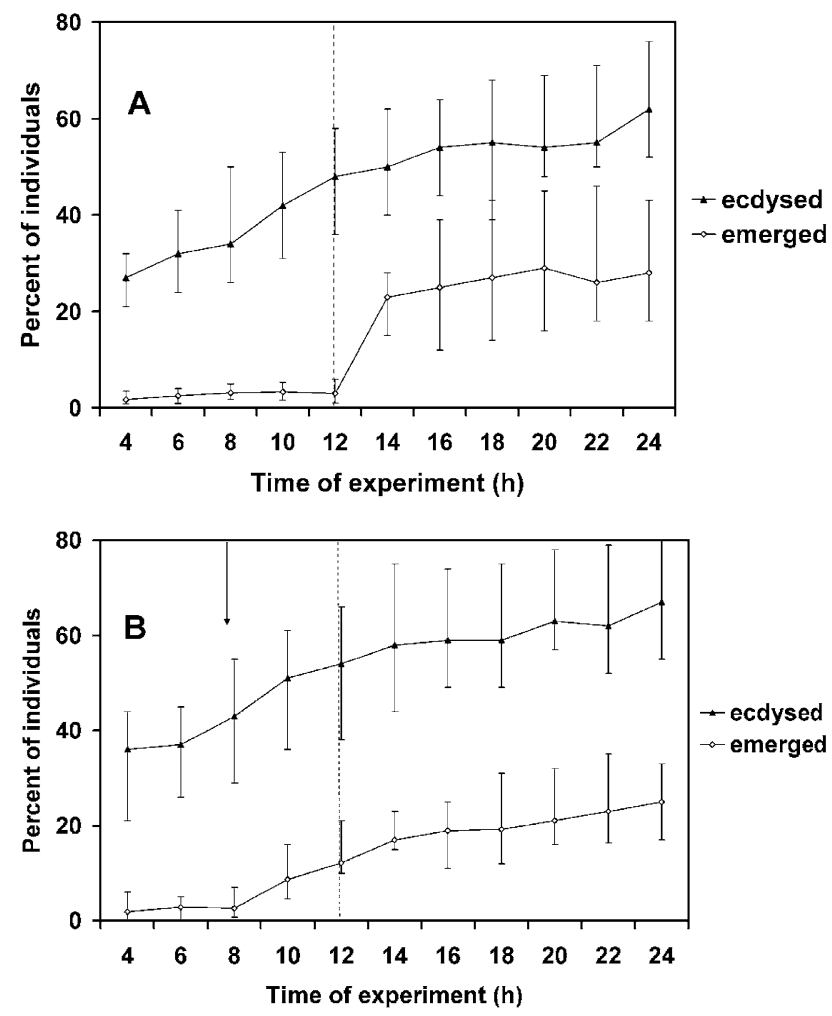

Fig. 2. Temporal patterns of adult ecdysis and emergence in Trichogramma embryophagum. Cumulative percentages of ecdysed and of emerged individuals (medians and quartiles) are shown, $\mathrm{n}=21$. A - the 2 nd experiment, vertical dashed line indicates the moment of lights-on. B - the 3rd experiment, vertical dashed line indicates the time of lights-on during the preceding development, black arrow indicates the advancement of the lights-on signal on the day of recording.

1A). Visual comparison showed that these individuals that remained inside the host chorion could easily be distinguished from just emerged adults by more transparent cuticle.

A more detailed study (the 2nd and the 3rd experiments) also did not reveal any diel rhythm of ecdysis. The cumulative percentage of ecdysed adults gradually increased with time, independently of whether the light was turned on in accordance with the entrained circadian rhythm or $4 \mathrm{~h}$ earlier, while the rhythm of emergence markedly changed (comp. Fig. 2A and 2B). Two-way ANOVA of pooled data of the 2nd and the $3 \mathrm{rd}$ experiments (the design of the experiment was the first factor and the circadian time was the second factor) showed that the ecdysis intensity was independent ( $p=$ 0.69) of the experimental design. The influence of the circadian time and the interaction of the two factors were also insignificant $(\mathrm{p}=0.10$ and $\mathrm{p}=0.63$, correspondingly).

The average emergence incidence was also independent of the design of the experiment $(\mathrm{p}=0.83)$, but it was strongly dependent on the circadian time $(\mathrm{F}=9.1, \mathrm{p}<$ $0.001)$. Noteworthy, the interaction was also highly significant $(\mathrm{F}=5.2, \mathrm{p}<0.001)$, proving that the patterns of time dependence in the 2 nd and the 3 rd experiments were different.

The average proportion of ecdysed adults that stayed in the host chorion after the peak of emergence in the 2nd experiment was $40 \%(32-45 \%)$, similar to that recorded after the main peak in the 1 st experiment.

\section{DISCUSSION}

The above results strongly suggest that the observed rhythm of Trichogramma adult emergence (Dahiya et al., 1993; Corrigan et al., 1995; Pompanon et al., 1995; Zaslavski et al., 1995, 1999; Karpova \& Reznik, 2002; Doyon \& Boivin, 2005; Karpova, 2006) is based exclusively (or at least mostly) on the rhythmic egression from the host egg chorion, not on the rhythmic pupal-adult ecdysis, although these two steps were not distinguished in the previous studies. Noteworthy, in most of the other studied insect taxa, adult ecdysis shows a robust circadian rhythm, although this rhythmicity could be based not only on the entrained circadian rhythm, but also on exogenous "masking" effects (Saunders, 2002). Arrhythmic adult ecdysis is very uncommon among free-living insects. It was observed in some blood-sucking mosquitoes (Haddow et al., 1959; Nayar, 1967; Jones \& Reiter, 1975). However, more detailed experiments with one of this species, Anopheles gambiae Giles, have finally revealed a circadian rhythm of eclosion (Reiter \& Jones, 1975). Some mutant lines and non-diapausing strains of flies Drosophila melanogaster Meigen (Konopka \& Benzer, 1971), Sarcophaga bullata Parker (Goto et al., 2006), and Chymomyza costata Zetterstedt (Lankinen \& Riihimaa, 1992) also show arrhythmic adult ecdysis, presumably caused by a malfunction of the circadian clock mechanism.

In Trichogramma, the arrhythmic ecdysis could be explained by the fact that, in contrast to many other insects, the ecdysed adults are within a well-protected space inside the host chorion and hence the exact timing of pupal-adult ecdysis is almost adaptively neutral. The timing of the next step, i.e., of the emergence from the host chorion is much more important. Synchronous eclosion of Trichogramma during the morning hours apparently minimizes the risk of desiccation and optimizes the reproductive activity of these parasitoids (Zaslavski et al., 1995, 1999; Karpova \& Reznik, 2002; Karpova, 2006). Interestingly, a similar distinct rhythm of emergence from the host was also observed in the braconid wasp Cotesia congregata (Say) that parasitizes larvae of the tobacco hornworm, Manduca sexta L. (Beckage et al., 2002). The parasitoid emerges from the host during the larval stage, the emergence coincides with the second larval ecdysis. This process is precisely timed and initiated almost invariantly during the photophase of 4 th or 5 th day after the host molts to the fifth instar larvae.

It should be mentioned that not only egg parasitoids, but many other endoparasitic hymenopterans pupate inside the dead hosts, host "mummies" or host puparia. In certain of these species, daily rhythms of adult emergence were observed (Mackauer \& Henkelman, 1975; 
Zinovjeva et al., 1996). However, in none of these studies were adult ecdysis and emergence investigated separately. Thus, it is possible that in some other endoparasitoids the observed daily rhythm of adult emergence is also based rather on the rhythmic egression from the host remains than on a rhythmic ecdysis.

We conclude that the ecdysed adults of Trichogramma stayed in the host chorion for a rather long time (Fig. 1A). Unfortunately, as was noted above, in only a few earlier studies with insect parasitoids were ecdysis and emergence separated (e.g. Teraoka \& Numata, 1995; Howard \& Baker, 2003). However, it was recorded that ecdysed Trichogramma adults may spend at least more than several hours within the host chorion (Dahlan \& Gordh, 1996). Moreover, in gregarious Trichogramma species, males ecdyse earlier than females but do not emerge from the chorion and thus mating occurs inside the empty host egg (Lee et al., 1988).

It seems that (at least in our experiments) this delay was not just waiting for the next "emergence gate". Even at the end of a peak of emergence the percentage of ecdysed adults that stayed in the chorion was rather high. The minimal time lag between ecdysis and emergence was about one day (Fig. 1A).

It is well known that newly eclosed adults have to undergo a teneral period before engaging in host search or other forms of activity. Particularly, the difference in chemical composition of cuticle between newly ecdysed and emerging adults of Habrobracon hebetor Say was revealed (Howard \& Baker, 2003). In our experiments, the individuals that stayed in the host chorion after the peak of mass emergence also differed from just emerged adults by having a more transparent cuticle. During the cuticular hardening, teneral adults are certainly very vulnerable to various adverse environmental factors and the host chorion could be used as a kind of temporary shelter. Thus it is conceivable that the ecdysed adults that have completed cuticular hardening, emerge during the first available "gate" while those, that have not, wait until a subsequent "gate".

ACKNOWLEDGEMENTS. This work was partly supported by the Programme of Department of Biological Sciences of RAS "Biological Resources of Russia: Fundamental Bases of Rational Use". We are thankful to T.S. Kats and T.Ya. Umarova for the excellent technical assistance. We are also deeply grateful to two anonymous reviewers for their comments and suggestions on an earlier version of the manuscript.

\section{REFERENCES}

BaKer J.D., McNabb S.L. \& Truman J.W. 1999: The hormonal coordination of behavior and physiology at adult ecdysis in Drosophila melanogaster. J. Exp. Biol. 202: 3037-3048.

Beckage N.E., Foreman R.C., Palmatier C.M. \& Tan F.F. 2002: Inhibition of the larval ecdysis and emergence behavior of the parasitoid Cotesia congregata by methoprene. J. Insect Physiol. 48: 725-732.

Corrigan J.E., Laing J.E. \& Zubricky J.S. 1995: Effects of parasitoid to host ratio and time of day of parasitism on development and emergence of Trichogramma minutum (Hymeno- ptera: Trichogrammatidae) parasitizing eggs of Ephestia kuehniella. Ann. Entomol. Soc. Am. 88: 773-780.

DAhiYa A.S., TShernyshev W.B. \& Afonina V.M. 1993: Diurnal rhythm of emergence from pupae in parasitic wasp. $J$. Interdiscipl. Cycle Res. 24: 162-170.

Dahlan A.N. \& GoRdH G. 1996: Development of Trichogramma australicum Girault (Hymenoptera: Trichogrammatidae) on Helicoverpa armigera (Hübner) eggs (Lepidoptera: Noctuidae). Austral. J. Entomol. 35: 337-344.

Doyon J. \& Boivin G. 2005: The effect of development time on the fitness of female Trichogramma evanescens. J. Insect Sci. 5: 4. Available online: insectscience.org/5.4.

Fantinou A.A., Alexandri M.P. \& Tsitsipis J.A. 1998: Adult emergence rhythm of the egg-parasitoid Telenomus busseolae. BioControl 43: 141-151.

Goto S.G., Han B. \& Denlinger D.L. 2006: A nondiapausing variant of the flesh fly, Sarcophaga bullata, that shows arrhythmic adult eclosion and elevated expression of two circadian clock genes, period and timeless. J. Insect Physiol. 52: 1213-1218.

Haddow A.J., Gillet J.D. \& Corbet P.S. 1959: Laboratory observations on pupation and emergence in the mosquito Aedes (Stegomyia) aegypti (L.). Ann. Trop. Med. Parasitol. 53: $123-131$.

HOWARD R.W. \& BAKER J.E. 2003: Cuticular hydrocarbons and wax esters of the ectoparasitoid Habrobracon hebetor: ontogenetic, reproductive, and nutritional effects. Arch. Insect Biochem. Physiol. 53: 1-18.

Jones M.D.R. \& Reiter P. 1975: Entrainment of the pupation and adult activity rhythms during development in the mosquito Anopheles gambiae. Nature 254: 242-244.

KARPOVA S.G. 2006: Role of endogenous and exogenous factors in the regulation of synchronous emergence of Trichogramma embryophagum Hartig and T. principium Sug. et Sor. (Hymenoptera, Trichogrammatidae). Entomol. Rev. 86: 252-263.

Karpova S.G. \& ReZnIK S.YA. 2002: Interaction of exogenous factors (light and temperature) in their influence on the daily pattern of adult eclosion in Trichogramma embryophagum (Hymenoptera: Trichogrammatidae). Eur. J. Entomol. 99: 427-436.

Konopka R.J. \& Benzer S. 1971: Clock mutants of Drosophila melanogaster. Proc. Nat. Acad. Sci. USA 68: 2112-2116.

Lankinen P. \& RiIHIMAa A.J. 1992: Weak circadian eclosion rhythmicity in Chymomyza costata (Diptera: Drosophilidae), and its independence of diapause type. J. Insect Physiol. 38: 803-811.

LeE K.Q., Jiang F.L. \& Guo J.J. 1988: Preliminary study of the reproductive behaviour of the parasitic wasps (Trichogramma). Colloques de l'INRA 43: 215-219.

Mackauer M. \& Henkelmann D.H. 1975: Effect of light-dark cycles on adult emergence in the aphids parasite Aphidius smithi. Can. J. Zool. 53: 1201-1206.

NAYAR J.K. 1967: The pupation rhythm in Aedes taeniorhynchus. II. Ontogenetic timing, rate of development, and endogenous diurnal rhythm of pupation. Ann. Entomol. Soc. Am. 67: 946-971.

Pompanon F., Fouillet P. \& Bouletreau M. 1995: Emergence rhythms and protandry in relation to daily patterns of locomotor activity in Trichogramma species. Evol. Ecol. 9: 467-477.

ReIRER P. \& Jones M.D.R. 1975: An eclosion timing mechanism in the mosquito Anopheles gambiae. J. Entomol. (A) 50: $161-168$. 
REYNOLDS S.E. 1980: Integration of behaviour and physiology in ecdysis. Adv. Insect Physiol. 15: 475-595.

SAUNDERS D.S. 2002: Insect Clocks. 3rd ed. Elsevier, Amsterdam, $560 \mathrm{pp}$.

SмITH S.M. 1996: Biological control with Trichogramma: advances, successes, and potential of their use. Annu. Rev. Entomol. 41: 375-406.

TERAoKa T. \& Numata H. 1995: Induction of adult diapause in a parasitoid wasp, Ooencyrtus nezarae under natural conditions. Entomol. Exp. Appl. 76: 329-332.

Zaslavski V.A., Zinovjeva K.B., Reznik S.Ya. \& Umarova T.YA. 1995: Effect of the photoperiod and thermoperiod on the eclosion rhythm in Trichogramma evanescens (Hymenoptera, Trichogrammatidae). Entomol. Exp. Appl. 74: 99-104.
Zaslavski V.A., Zinovjeva K.B., Umarova T.Ya. \& RezniK S.YA. 1999: Interaction of circadian rhythm synchronised by photoperiod and by thermoperiod with direct influence of light and temperature as factors determining rhythm of adult eclosion in two species of Trichogramma (Hymenoptera, Trichogrammatidae). Entomol. Rev. 79: 1-10.

Zinovjeva K.B., Reznik S.Ya., Zaslavski V.A. \& Umarova T.YA. 1996: Effect of photoperiod and thermoperiod on the eclosion rhythm of adults of Alysia manducator (Hymenoptera, Braconidae) and Trichogramma evanescens (Hymenoptera, Trichogrammatidae). Entomol. Rev. 76: 1244-1250.

Received June 26, 2007; revised and accepted July 16, 2007 